01.3

\title{
Влияние на корреляционные характеристики переключений между разными хаотическими режимами
}

\author{
(C) О.Н. Павлова ${ }^{1}$, А.Н. Павлов ${ }^{1,2, \text { ฯ }}$ \\ ${ }^{1}$ Саратовский национальный исследовательский государственный университет им. Н.Г. Чернышевского, \\ Саратов, Россия \\ ${ }^{2}$ Саратовский государственный технический университет им. Гагарина Ю.А., Саратов, Россия \\ ฯE-mail: pavlov.alexeyn@gmail.com
}

Поступило в Редакцию 20 мая 2019г.

В окончательной редакции 20 мая 2019г.

Принято к публикации 3 июня 2019г.

Рассматривается задача диагностики режимов динамики нелинейных систем по экспериментальным данным при наличии переключений между колебательными процессами. Исследуется влияние ошибок вычисления корреляционных характеристик, вызванных присутствием фрагментов данных, соответствующих разным динамическим режимам, на интерпретацию результатов. Показано, что переключения между режимами функционирования значительно сильнее влияют на диагностику антикоррелированной динамики.

Ключевые слова: сигнал, перемежаемость, корреляционный анализ, хаотическая динамика.

DOI: 10.21883/PJTF.2019.18.48228.17882

Диагностика режимов динамики нелинейных систем по экспериментально регистрируемым сигналам часто осложняется наличием сбойных участков, помех различной природы и изменением во времени характеристик системы, приводящим к необходимости анализировать участки небольшой длительности [1-3]. Предварительная обработка данных позволяет выявлять и устранять хорошо различимые артефакты [4-6] путем использования методов фильтрации, применимых к сигналам с локализованными помехами [7-10], или простого удаления сбойных фрагментов. Такие подходы широко используются во многих экспериментальных исследованиях, например, в нейрофизиологии, где доля данных, содержащих артефакты, часто превосходит длительность записи, пригодной для анализа, и соответствующие участки обычно устраняются из дальнейшего рассмотрения $[11,12]$. Однако при этом меняются корреляционные характеристики [13-15], что необходимо учитывать при решении задач диагностики. Но если сбойные фрагменты, как правило, хорошо различимы даже при визуальном контроле экспериментальных данных, то изменения во времени характеристик системы при переключениях между режимами функционирования часто оказываются незаметными. В этом случае обработка соответствующих сигналов проводится в ошибочном предположении постоянства параметров, результатом чего является неправильная интерпретация вычисляемых величин.

В настоящей работе анализируется влияние переключений между колебательными режимами различной сложности на результаты расчета корреляционных характеристик. Мы покажем, как ошибки вычисления, вызванные присутствием в сигнале фрагментов колебательных процессов, соответствующих разным дина- мическим режимам, сказываются на интерпретации полученных результатов, и отметим их роль для коррелированной и антикоррелированной динамики систем различной природы. В качестве исследуемых систем выбрано несколько базовых моделей нелинейной динамики, демонстрирующих режимы хаотических или гиперхаотических колебаний. Дополнительно рассмотрены переходные процессы в динамике сердечно-сосудистой системы.

Поскольку изменение режимов функционирования системы в общем случае приводит к нестационарным процессам, в качестве метода исследования был выбран один из вариантов флуктуационного анализа (detrended fluctuation analysis, DFA), который относится к числу наиболее универсальных подходов, применимых как к стационарным, так и к нестационарным временны́м рядам [16-18]. Этот метод включает переход от исходного сигнала $x(k), k=1, \ldots, N$ к его профилю $Y(i)=\sum_{k=1}^{i}[x(k)-\langle x\rangle]$ (где $\langle x\rangle-$ среднее значение), деление профиля на неперекрывающиеся сегменты длины $n$ и расчет среднеквадратичного отклонения $F(n)$ профиля $Y(i)$ от его линейной аппроксимации $Y_{n}(i)$ в пределах каждого сегмента [17]. Зависимость среднеквадратичного отклонения от длины сегмента описывается степенным законом вида $F(n) \sim n^{\alpha}$, где величина $\alpha$ (показатель скейлинга метода DFA) отражает коррелированную $(\alpha>0.5)$ и антикоррелированную $(\alpha<0.5)$ динамику и связана с показателями, характеризующими спад автокорреляционной функции и частотную зависимость спектральной плотности мощности [16].

Рассмотрим применение этого метода для анализа переключений между колебательными процессами в модели двух связанных систем Ресслера, которая опи- 
сывается следующими уравнениями:

$$
\begin{gathered}
\frac{d x_{1,2}}{d t}=-\omega_{1,2} y_{1,2}-z_{1,2}+\gamma\left(x_{2,1}-x_{1,2}\right) \\
\frac{d y_{1,2}}{d t}=\omega_{1,2} x_{1,2}+a y_{1,2}, \quad \frac{d z_{1,2}}{d t}=b+z_{1,2}\left(x_{1,2}-c\right),
\end{gathered}
$$

где выбраны значения параметров $\omega_{1,2}=1.0 \pm \Delta$, $a=0.15, b=0.2, \gamma=0.02$, а параметры $\Delta$ и $c$ варьировались для перехода между различными режимами регулярной, хаотической и гиперхаотической динамики [19]. Сначала были изучены переключения между синхронным хаосом $\left(C A_{s y n}, c=6.8, \Delta=0.0096\right)$ и несинхронным хаосом $\left(C A_{a s y n}, c=6.8, \Delta=0.0098\right)$. Эти режимы колебаний, анализируемые по последовательностям времен возврата в секущую Пуанкаре $x_{2}+y_{1}=0$, описываются соответственно значениями показателя скейлинга $\alpha \approx 0.02$ и $\approx 0.80$. Антикоррелированная структура последовательностей времен возврата синхронного режима $C A_{s y n}$ является следствием субгармонического каскада, приводящего к формированию хаотического аттрактора [19], тогда как режим несинхронного хаоса $C A_{\text {asyn }}$ возникает при разрушении квазипериодических колебаний [19] и демонстрирует коррелированную структуру последовательностей времен возврата. Если анализируемые сигналы содержат переходы между режимами $C A_{s y n}$ и $C A_{a s y n}$ (мы рассматривали случай случайных переключений, при котором общая длительность фрагментов данных каждого режима совпадает), то это приводит к величине $\alpha \approx 0.63$. Таким образом, смещение показателя скейлинга является несимметричным: для антикоррелированной динамики оно примерно в 3.5 раза больше, чем для коррелированной.

Отметим, что этот вывод подтверждается и для других вариантов переключений между режимами с коррелированными и антикоррелированными последовательностями времен возврата, например для случайных переключений между гиперхаосом (HA, $c=7.2$, $\Delta=0.0094$, антикоррелированная динамика с $\alpha \approx 0.03$ ) и несинхронным хаосом $\left(C A_{a s y n}, c=7.2, \Delta=0.0097\right.$, коррелированная динамика с показателем $\alpha \approx 0.79)$ или для переключений между циклами больших периодов и квазипериодическими колебаниями. При этом переходы между режимами коррелированной динамики или между режимами антикоррелированной динамики не сопровождались существенными смещениями показателя скейлинга. Данное обстоятельство означает, что наибольшую сложность для диагностики колебательных процессов по экспериментальным данным вызывает случай переключений между колебаниями с коррелированной и антикоррелированной статистикой времен возврата. Выше была рассмотрена одинаковая длительность сигналов. На рис. 1 показано, как меняется показатель скейлинга, если один из режимов является основным (система проводит в этом режиме бо́льшую часть времени), а сравнительная часть времени пребывания во втором режиме варьируется. Если в качестве основного рассматривается
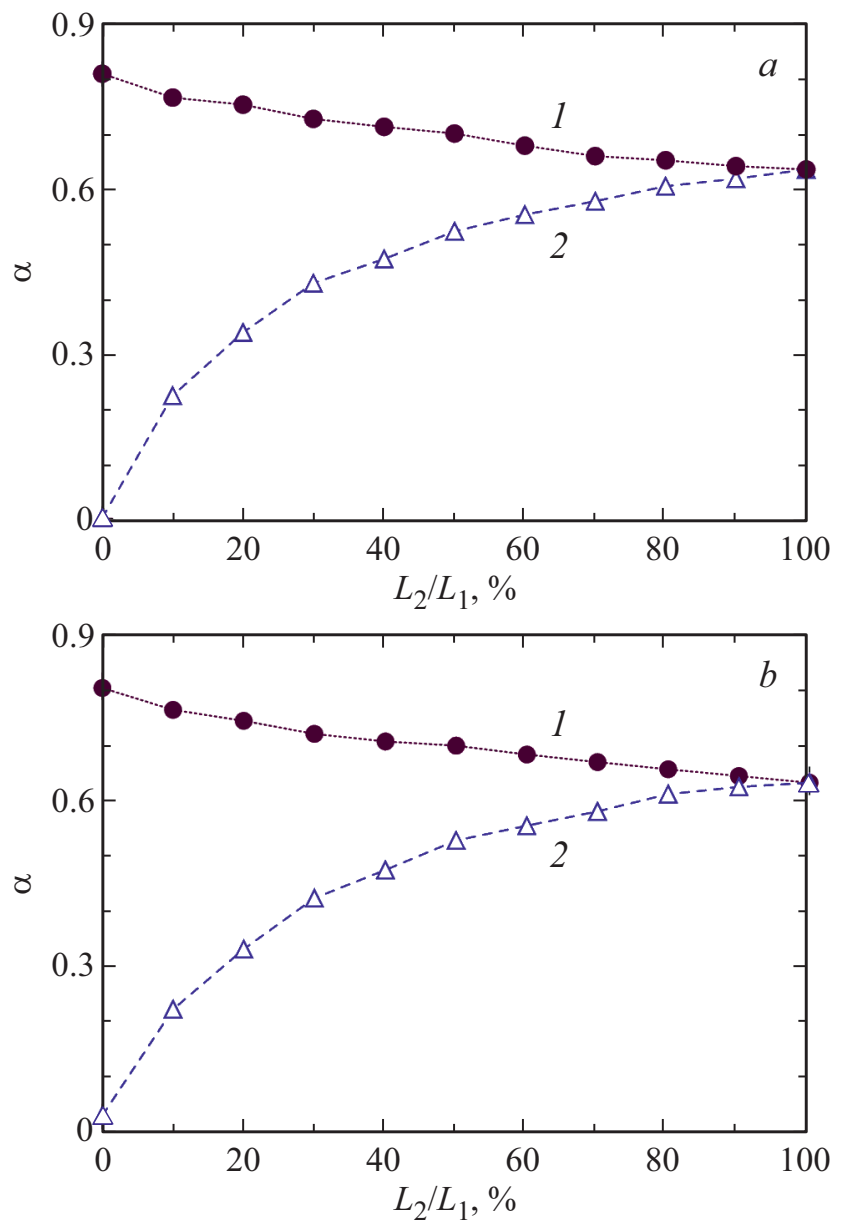

Рис. 1. Изменение показателя скейлинга $\alpha$ режимов динамики двух связанных систем Ресслера при наличии переключений между синхронными и несинхронными хаотическими колебаниями $(a)$ и между гиперхаосом и несинхронным хаосом $(b)$. Значения $L_{1}$ и $L_{2}$ определяют суммарную длительность участков данных основного и неосновного режима динамики. Показаны результаты, полученные при выборе в качестве основного режима колебаний с коррелированными (1) и антикоррелированными (2) последовательностями времен возврата в сечение Пуанкаре.

режим антикоррелированной динамики, то появление участков данных с коррелированной статистикой времен возврата приведет к смещению показателя скейлинга, которое в несколько раз превышает соответствующее смещение, происходящее в случае выбора режима коррелированной динамики в качестве основного (рис. 1).

Для подтверждения сделанного вывода были рассмотрены хаотические колебания в функционировании двух связанных систем Лоренца

$$
\begin{gathered}
\frac{d x_{1,2}}{d t}=\sigma\left(y_{1,2}-x_{1,2}\right)+\gamma\left(x_{2,1}-x_{1,2}\right), \\
\frac{d y_{1,2}}{d t}=r_{1,2} x_{1,2}-x_{1,2} z_{1,2}-y_{1,2}, \frac{d z_{1,2}}{d t}=x_{1,2} y_{1,2}-z_{1,2} b
\end{gathered}
$$

с параметрами $\sigma=10, r_{1}=28.8, r_{2}=28.0 . \quad b=8 / 3$. Переключения параметра $\gamma$ между значениями $\gamma=2.0$ 


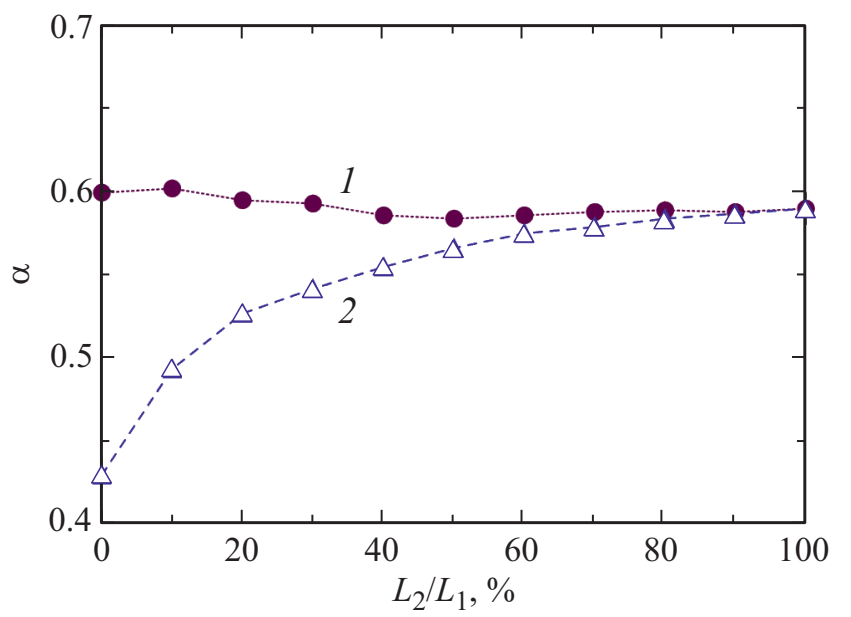

Рис. 2. Изменение показателя скейлинга $\alpha$ режимов динамики двух связанных систем Лоренца при наличии переключений между синхронными и несинхронными хаотическими колебаниями. Обозначения соответствуют приведенным на рис. 1.

и 8.0 приводили к переходам между режимами с коррелированной $(\gamma=2.0, \alpha \approx 0.60)$ и антикоррелированной $(\gamma=8.0, \alpha \approx 0.43)$ статистикой времен возврата в секущую Пуанкаре $z_{1}=30$. На рис. 2 показано, что в этом случае антикоррелированная динамика также намного чувствительнее к присутствию участков, соответствующих неосновному режиму колебаний.

Дополнительно были рассмотрены экспериментальные данные, содержащие переходные процессы в реакции сердечно-сосудистой системы на стресс. В качестве измеряемого сигнала была выбрана временна́я зависимость артериального давления крови крысы. В соответствии с нашими предыдущими исследованиями [20] для таких сигналов типична коррелированная динамика при различных функциональных состояниях организма. Тем не менее встречаются нетипичные примеры, когда стресс вызывает переход к антикоррелированной динамике. Для одного из таких примеров были проанализированы переходы между состоянием с $\alpha \approx 0.93$ и состоянием с $\alpha \approx 0.45$. Участки записи, соответствующие этим состояниям, варьировались по аналогии с модельными системами. Проведенные расчеты показали, что при выборе антикоррелированной динамики в качестве основного режима смещение показателя скейлинга, вызванное присутствием участков коррелированного поведения, в несколько раз больше, чем в случае, когда основной является коррелированная динамика. Таким образом, все рассмотренные примеры свидетельствуют о том, что на диагностику антикоррелированной динамики значительно сильнее влияют переключения между режимами функционирования, которые могут присутствовать в регистрируемых экспериментальных данных.

\section{Финансирование работы}

Исследование выполнено при поддержке гранта Российского научного фонда (проект № 19-12-00037).

\section{Соблюдение этических стандартов}

Все применимые международные, национальные и/или институциональные принципы ухода и использования животных были соблюдены.

\section{Конфликт интересов}

Авторы заявляют, что у них нет конфликта интересов.

\section{Список литературы}

[1] Bendat J.S., Piersol A.G. Random data: analysis and measurement procedures. 4th ed. N.J.: Wiley, 2010. 640 p.

[2] Addison P.S. The illustrated wavelet transform handbook: introductory theory and applications in science, engineering, medicine and finance. 2nd ed. Boca Raton: CRC Press, 2016. $464 \mathrm{p}$.

[3] Chatfield $C$. The analysis of time series: an introduction. 6th ed. Boca Raton: Chapman \& Hall / CRC, 2003. 352 p.

[4] Benesty J., Chen J. A conceptual framework for noise reduction. Berlin: Springer, 2015. $89 \mathrm{p}$.

[5] Loizou P.C. Speech enhancement. Theory and practice. 2nd ed. Boca Raton: CRC Press, 2013. 711 p.

[6] Selesnick I.W., Baraniuk R.G., Kingsbury N.G. // IEEE Signal Process. Mag. 2005. V. 22. N 6. P. 123-151.

[7] Ding Y., Selesnick I.W. // IEEE Signal Process. Lett. 2015. V. 22. N 9. P. $1364-1368$.

[8] Ясин А.С., Павлов А.Н. // Письма в ЖТФ. 2015. Т. 41. В. 14. C. 33-38.

[9] Ясин А.С., Павлова О.Н., Павлов А.Н. // Письма в ЖТФ. 2016. T. 42. B. 2. C. $50-56$.

[10] Ясин А.С., Павлова О.Н., Павлов А.Н. // Письма в ЖТФ. 2017. T. 43. B. 14. C. 10-18.

[11] Urigüen J.A., Garcia-Zapirain B. // J. Neural. Eng. 2015. V. 12. N 3. P. 031001.

[12] Zhang C., Tong L., Zeng Y., Jiang J., Bu H., Yan B., Li J. // BioMed Res. Int. 2015. V. 2015. P. 720450.

[13] Ma Q.D.Y., Bartsch R.P., Bernaola-Galván P., Yoneyama M., Ivanov P.Ch. // Phys. Rev. E. 2010. V. 81. N 3. P. 031101.

[14] Pavlov A.N., Pavlova O.N., Abdurashitov A.S., Sindeeva O.A., Semyachkina-Glushkovskaya O.V., Kurths J. // Chaos. 2018. V. 28. N 1. P. 013124.

[15] Павлова О.Н., Павлов А.Н. // Письма в ЖТФ. 2018. Т. 44. B. 14. C. $80-85$.

[16] Peng C.-K., Buldyrev S.V., Havlin S., Simons M., Stanley H.E., Goldberger A.L. // Phys. Rev. E. 1994. V. 49. N 2. P. 1685-1689.

[17] Peng C.-K., Havlin S., Stanley H.E., Goldberger A.L. // Chaos. 1995. V. 5. N 1. P. 82-87.

[18] Bryce R.M., Sprague K.B. // Sci. Rep. 2012. V. 2. P. 315.

[19] Postnov D.E., Vadivasova T.E., Sosnovtseva O.V., Balanov A.G., Anishchenko V.S., Mosekilde E. // Chaos. 1999. V. 9. N 1. P. 227-232.

[20] Pavlov A.N., Ziganshin A.R., Klimova O.A. // Chaos Solit. Fractals. 2005. V. 24. N 1. P. 57-63. 